\title{
Effect of blanching on enzyme activity, color changes, anthocyanin stability and extractability of mangosteen pericarp: A kinetic study
}

\begin{abstract}
Mangosteen pericarp is a rich source of anthocyanins. However, the high polyphenol oxidase (PPO) activity challenges potential applications of pericarp as natural colorant. The kinetics of PPO inactivation, anthocyanin loss and color changes were determined over a temperature range of $60-100{ }^{\circ} \mathrm{C}$. First-order kinetic model provided the best prediction of the PPO inactivation $\left(R^{2} \geq 0.977\right)$, while anthocyanin loss was described by a Weibull kinetic model $\left(R^{2} \geq 0.969\right)$. The activation energies of PPO inactivation, anthocyanin loss and total color changes were $43.11,57.66$ and $18.86 \mathrm{~kJ} / \mathrm{mol}$, respectively. Anthocyanin content was the most sensitive parameter towards temperature changes, suggesting the importance of its monitoring as a quality parameter during thermal processing. Blanching enhanced the efficiency of anthocyanin extraction. Gathering the quantitative information on the changes of PPO activity as well as quality characteristics during blanching of mangosteen pericarp is important in order to design a proper pre-processing condition.
\end{abstract}

Keyword: Mangosteen pericarp; Anthocyanin; Polyphenol oxidase; Kinetics; Weibull model; First order 